\title{
$\begin{array}{ll}\text { Research Square } & \text { Preprints are preliminary reports that have not undergone peer review. } \\ \text { They should not be considered conclusive, used to inform clinical practice, }\end{array}$ or referenced by the media as validated information. \\ Radical Hysterectomy Versus Simple Hysterectomy and Brachytherapy for Patients with Stage II Endometrial Cancer
}

\section{Ming Wang}

Department of Gynecologic Oncology, Beijing Obstetrics and Gynecology Hospital, Capital Medical University

\section{Ran Ran}

Department of Obstetrics and Gynecology, Beijing Youan Hospital, Capital Medical University Yu-Mei Wu ( $\square$ wym597118@163.com )

Beijing Obstetrics and Gynecology Hospital, Capital Medical University

\section{Research}

Keywords: Endometrial cancer, Postoperative radiation, Propensity score matching, Survival

Posted Date: December 3rd, 2020

DOI: https://doi.org/10.21203/rs.3.rs-117737/v1

License: (c) (i) This work is licensed under a Creative Commons Attribution 4.0 International License. Read Full License 


\section{Abstract}

\section{Purpose}

To compare the survival outcome between radical hysterectomy and total hysterectomy with radiation therapy in patients with stage II endometrial cancer.

\section{Methods}

This is a retrospective cohort study. We identified 1349 patients diagnosed with stage II endometrial cancer from Jan 1, 1988 to Dec 312015 in the Surveillance, Epidemiology, and End Results. Patients were divided into two groups based on the primary treatment (total hysterectomy combined with brachytherapy or radical hysterectomy). All patients received external beam radiation therapy after the surgery. The primary outcome was the rate of 5-year-cause-specific survival and 5-year-overall survival.

\section{Results}

A total of 1349 patients were enrolled in the study, 117(7.35\%) patients received radical hysterectomy and 460 patients who received total hysterectomy combined with vaginal brachytherapy were selected as control. All patients received external beam radiation therapy after the surgery. Overall, the median followup duration was $82.77 \pm 1.44$ months ( $95 \% \mathrm{Cl}$ : $79.94-85.61$ months). There was no difference in the baseline information between two groups, including ages, ethnicity, and rates of histologic subtypes. The 5-year overall survival was $62.31 \%$ among women who underwent radical hysterectomy which was lower than $78.48 \%$ among those who underwent total hysterectomy combined with vaginal brachytherapy (HR, 2.22; $95 \% \mathrm{Cl}, 1.52$ to $3.24 ; \mathrm{P}<0.001$ by the log-rank test). Women who underwent radical hysterectomy also had shorter 5-year cause-specific survival $(74.60$ vs.85.38\%; $\mathrm{HR}, 1.91 ; 95 \% \mathrm{Cl}, 1.13$ to $3.23 ; \mathrm{P}=0.01$ by the logrank test) than those who underwent total hysterectomy combined with vaginal brachytherapy. However, the negative outcomes were further validated in patients with high-risk endometrial cancer, not in patients with grade 1-2 low-risk endometrial cancer both on cause-specific survival and overall survival. In patients with grade 3 low-risk endometrial cancer, the tendency was only found with lower overall survival not cause-specific survival.

\section{Conclusions}

This study revealed that in patients' stage II endometrial cancer, radical hysterectomy was associated with shorter overall survival and cause-specific survival than total hysterectomy combined with vaginal brachytherapy. The choice of different treatment modalities should base on the histology subtype of patients and further study based on molecular classification is needed.

\section{Introduction}

The incidence and mortality rates of endometrial cancer $(\mathrm{EC})$ remain high in developed countries and are increasing among women in developing countries [1, 2]. An estimated 382069 new cases of EC were 
diagnosed around the world in 2018, and 89929 people died of the disease. As a general principle, EC should be removed en bloc to optimize outcomes and intraperitoneal morcellation should be avoided [3]. For patients with suspected or gross cervical involvement (endometrioid histology), total hysterectomy (TH) or radical hysterectomy is recommended along with bilateral salpingo-oophorectomy (BSO), cytology (peritoneal lavage), and dissection of lymph nodes if indicated [4]. In these patients, radical or modified radical hysterectomy may improve local control and survival when compared with $\operatorname{TH}[5,6]$. Alternatively, the patient may undergo external beam radiation therapy (EBRT) and vaginal brachytherapy (BT) (category 2B) followed by $\mathrm{TH} / \mathrm{BSO}$ and surgical staging. For patients with stage Il disease who have had a radical hysterectomy with negative surgical margins and no evidence of extrauterine disease, observation or EBRTs are options.

To date, there is no better choice due to limited comparative studies between the outcomes of two modalities. In developing countries, especially the radiation equipment not widely used, the radical hysterectomy may be a better choice. For stage II EC patients who received radical hysterectomy with a negative surgical margin and no evidence of extrauterine disease, radiotherapy could be avoided in low and intermediate-risk patients [7]. A systematic review included 10 retrospective cohort studies enrolling 2866 patients. Patients who received radical hysterectomy did not show a significant survival benefit for either overall survival(OS) or progress free survival. Radical hysterectomy showed a $27 \%$ survival benefit (pooled Hazard ratio(HR) 0.73; 95\% $\mathrm{Cl} 0.53-1.00 ; \mathrm{P}=0.050$ ) in earlier studies based on Federation International of Gynecology and Obstetrics(FIGO) 1988 staging, whereas showed increased risk of death (pooled HR 1.24; 95\% Cl 0.86-1.77; P = 0.245) in newly published studies based on FIGO 2009 staging [8]. However, all have no statistical significance. The reasons underlying this are still unclear. Surgeons may tend to perform radical hysterectomy in more severe cases with poorer prognostic factors. The advances in radiotherapy may lower the risk of pelvic or vaginal recurrence resulting from insufficient parametrial margins in $\mathrm{TH}$.

This study has aimed to evaluate the survival of different treatment models in patients with EC involved cervix. We conducted a retrospective cohort study of 577 patients to give a more complete picture of the real-world clinical outcomes to enhance the body of different treatment modalities.

\section{Materials And Methods}

\section{Study design and patient selection}

This study was a retrospective study involving data from patients' stage II EC, registered in the Surveillance, Epidemiology, and End Results (SEER) registry (Third Edition, SEER 18 registry database November 2019 submission) [9] from 1988 to 2015. This database covers approximately $27.8 \%$ of the U.S. population and is publicly available and de-identified. The data reported in this study represent the most recent follow-up (April 30, 2019) available in the SEER database. 
Patients diagnosed between Jan 1, 1988, and Dec 31, 2015, with primary EC involving cervix treated with radical hysterectomy or $\mathrm{TH}$ combined with vaginal $\mathrm{BT}$ were eligible for participation. All patients received EBRT after the surgery. Patients who didn't undergo radiation therapy (RT) for any reason were excluded, and we also excluded patients if we could not determine whether they matched the inclusion criteria because of missing data (e.g., surgery information or postoperative radiotherapy).

\section{Study Procedures and Data Collections}

SEER*Stat 8.2.3 was used to extract the data and women fulfilling the aforementioned enrollment criteria were offered participation in the study. Detailed demographic, oncological, and survival data were collected. We divided our cohort into two groups according to treatment modalities: TH (group A) combined with vaginal BT and radical hysterectomy (group B). Group A was considered having postoperative with vaginal BT after TH with Federation International of Gynecology and Obstetrics (FIGO) stage II EC, Group B contained patients with FIGO stage II EC and didn't receive vaginal BT, all patients received EBRT after the surgery. The cancer stage was reclassified into FIGO 2019, based on tumor size, tumor extension, and lymph node status recorded in the database.

Propensity score matching for each group was computed for each case determined by multivariable logistic regression analysis. Patient demographics, tumor characteristics, and treatment patterns were entered in the propensity score model. Four-to-one propensity score matching between Group A and Group B was performed through an automated algorithm with the propensity score difference cut off being $1 \%$.

\section{Outcome Measurements}

Survival data, including 5-year-cause-specific survival (CSS) and 5-year-OS (all-cause mortality), are collected through linkages with state mortality records and the National Death Index. CSS was defined as the time interval between the initial diagnosis of uterine EC and the date of death resulting from this specific disease. OS was defined as the time interval between the initial uterine EC diagnosis and the date of death for any reason. Among women who died, causes of death were examined (uterine EC and other diseases) and grouped as previously described.

The primary outcome was to examine the 5-year-OS among women in the two groups, respectively. The secondary outcome was to examine the 5-year-CSS in the two groups, respectively.

\section{Statistical analysis}

Rank sum test or $\chi 2$ test was used to exam base-line characteristics: age at diagnosis, ethnicity, year at diagnosis, grade, surgery modality, postoperative radiation, chemotherapy. Cox regression was used to evaluate proportional hazard regression models, and the magnitude of statistical significance was expressed with a hazard ratio (HR) and $95 \% \mathrm{Cl}$.

We employed Kaplan-Meier analysis to construct survival and cumulative risk curves, and statistical significance between the curves was compared with log-rank tests. Survival was also examined using 
Cox Covariates, entered in the final model were patient demographics, tumor factors, and treatment patterns.

Statistical analyses were conducted using SPSS23. All P-values reflected 2-sided tests, and significance was set at $<0.05$.

\section{Results}

\section{Study Population}

A total of 1718 patients were screened and 1349 patients were enrolled in the study from 1988 to 2015 (Fig. 1). All patients were found with cervical stromal invasion and received cancer-directed hysterectomy and postoperative EBRT. 117(7.35\%) patients received radical hysterectomy and 460 patients who received TH combined with vaginal BT were selected as control. The baseline characteristics of the patients are summarized in Table 1. The mean age of the patients in group $A$ was comparable to the patients in group $B(61.27 \pm 12.34$ vs.61.39 \pm 11.80 years, $p=0.92)$. There were no significant differences between the two groups with respect to age $(p=0.96)$, ethnicity $(P=0.06)$, year at diagnosis $(P=0.06)$, grade $(P=0.63)$, chemotherapy $(p=0.51)$. 
Table 1

Clinicopathologic and Treatment Characteristics of Study Patients

\begin{tabular}{|c|c|c|c|}
\hline Values & $\begin{array}{l}\text { Group A } \\
(n=117)\end{array}$ & $\begin{array}{l}\text { GroupB } \\
(n=460)\end{array}$ & P-value \\
\hline Age & $61.27 \pm 12.34$ & $61.39 \pm 11.80$ & $P=0.92$ \\
\hline$<45$ & $10(8.5)$ & $36(7.8)$ & \multirow[t]{5}{*}{$P=0.96$} \\
\hline $45-55$ & 28(23.9) & $113(24.6)$ & \\
\hline $55-65$ & $35(29.9)$ & $140(30.4)$ & \\
\hline $65-75$ & $27(23.1)$ & $115(25.0)$ & \\
\hline$>=75$ & $17(14.5)$ & $56(12.2)$ & \\
\hline \multicolumn{4}{|l|}{ Ethnicity } \\
\hline White & 84(71.8) & $359(78.0)$ & \multirow[t]{3}{*}{$P=0.06$} \\
\hline Black & 16(13.7) & $32(7.0)$ & \\
\hline Other & $17(14.5)$ & $69(15.0)$ & \\
\hline \multicolumn{4}{|l|}{ Year at diagnosis } \\
\hline $1998-2000$ & $14(12.0)$ & $70(15.2)$ & \multirow[t]{4}{*}{$P=0.06$} \\
\hline $2001-2005$ & $25(21.4)$ & $147(32.0)$ & \\
\hline $2006-2010$ & $38(32.5)$ & 118(25.7) & \\
\hline $2011-2015$ & $40(34.2)$ & $125(27.2)$ & \\
\hline \multicolumn{4}{|l|}{ Grade } \\
\hline Grade 1-2 low-risk endometrial cancer & $48(41.0)$ & 211(45.9) & \multirow[t]{3}{*}{$P=0.63$} \\
\hline Grade 3 low-risk endometrial cancer & 46(39.3) & 163(35.4) & \\
\hline High-risk endometrial cancer & 23(19.7) & $86(18.7)$ & \\
\hline \multicolumn{4}{|l|}{ Chemotherapy } \\
\hline No & $83(70.9)$ & $324(70.4)$ & \multirow[t]{2}{*}{$P=0.51$} \\
\hline Yes & $34(29.1)$ & 136(29.6) & \\
\hline
\end{tabular}

\section{Impact of different treatment modalities on survival}

The median follow-up time was $82.77 \pm 1.44$ months (95\%Cl: $79.94-85.61$ months). Figure 2 shows the results of Kaplan-Meier analyses of 5-year OS (Fig. 2A) and 5-year-CSS (Fig. 2B) associated with different treatment modalities. Women who underwent radical hysterectomy had shorter 5-year OS $(62.31 \%$ 
vs.78.48\%; HR, 2.22; $95 \% \mathrm{Cl}, 1.52$ to $3.24 ; \mathrm{P}<0.001$ by the log-rank test) and 5-year-CSS (74.60 vs.85.38\%; $\mathrm{HR}, 1.91 ; 95 \% \mathrm{Cl}, 1.13$ to $3.23 ; \mathrm{P}=0.014$ by the log-rank test).) than those who underwent $\mathrm{TH}$ combined with vaginal BT. To better know the effect of pathological type on survival, we further compared the survival outcomes in patients with grade 1-2 low-risk EC, grade 3 low-risk EC, and high-risk EC, independently. Women who underwent radical hysterectomy had shorter OS (53.39\% vs. $70.99 \%$; HR, 2.12; $95 \% \mathrm{Cl}, 1.19$ to $3.77 ; \mathrm{P}=0.0018$ by the log-rank test) and CSS $(62.53 \%$ vs.78.79\%; $\mathrm{HR}, 1.90 ; 95 \% \mathrm{Cl}, 0.90$ to 3.38; $\mathrm{P}=0.044$ by the log-rank test) in patients with high-risk EC. However, the same results were not found in patients with grade $1-2$ low risk EC both on CSS (75.29\% vs. $69.44 \% ; \mathrm{HR}, 1.06 ; 95 \% \mathrm{Cl}, 0.42$ to $2.66 ; \mathrm{P}=0.90$ by the log-rank test) and $\mathrm{OS}(75.38 \%$ vs. $90.05 \% ; \mathrm{HR}, 1.15 ; 95 \% \mathrm{Cl}, 0.52$ to $1.98 ; \mathrm{P}=0.96$ by the log-rank test). Grade 3 low risk EC were only found with lower OS (75.38\% vs. $90.05 \% ; H R, 2.34 ; 95 \%$ $\mathrm{Cl}, 1.16$ to $4.70 ; \mathrm{P}=0.0012$ by the log-rank test) not CSS ( $88.84 \%$ vs. $94.09 \% ; \mathrm{HR}, 2.04 ; 95 \% \mathrm{Cl}, 0.64$ to $6.45 ; P=0.12$ by the log-rank test).(Fig. 3 )

\section{Discussion}

The present study compared two different treatment modalities of stage II EC and found that radical hysterectomy without vaginal BT was associated with a higher risk of death than women who underwent TH combined with vaginal BT for stage II EC.

The optimal treatment for patients with FIGO 2009 Stage II disease (those harboring cervical stromal invasion) is uncertain and is debated [10]. Sartori et al evaluated 203 patients with stage II EC and compared patients who underwent a $\mathrm{TH}$ and radical hysterectomy. They found a significant improvement in 5 -year OS that was $94 \%$ in the radical hysterectomy group versus $79 \%$ in the $\mathrm{TH}$ group ${ }^{7}$. However, a recent study published by Takano et al evaluated 300 patients with stage II EC, they found that the type of hysterectomy was not an independent prognostic factor for Disease-free survival and OS. Besides, they reported that perioperative and late adverse events were more common in patients treated with radical hysterectomy. Similar results were observed by other investigators [11-13]. Although surgical resection is the mainstay of treatment for patients with early-stage EC, randomized studies have shown that adjuvant radiation treatment for patients with early-stage disease and high-risk factors reduced rates of local recurrence $[14,15]$, such as age $\geq 60$ yrs., deep myoinvasion (> 50\%), lymph vascular invasion (LVSI) positive, and high-grade histology decreased rates of local recurrence. The use of vaginal BT allows for the delivery of localized radiation to the vaginal cuff which reduces the localized recurrence of TH. In previous studies in patients with stage II EC [16, 17], no difference was found between TH followed by both whole pelvic and vaginal BT or by radical hysterectomy alone. However, these results are biased by heterogeneity and small sample sizes. And the staging system from FIGO 2009 had a different definition of Stage II EC.

In our study, we found that in the case of external irradiation in both groups, the survival period of TH plus vaginal BT was higher than that of the radical operation group. However, the negative outcomes were further validated in patients with high-risk EC, not in patients with grade 1-2 low-risk EC both on causespecific survival and OS. Grade 3 low-risk EC was only found with lower OS, not CSS. The different results 
in low-risk grade 1-2 patients indicated that other reasons undying the death of this population. Now endometrial cancers were classified into four categories: POLE-mutated (POLE-mt), mismatch-repairdeficient (MMR-d), p53-abnormal (p53abn), p53-wild-type (p53wt) [18]. The p53-abn group showed the worst prognosis of four molecular types. Prevalence of high-risk factors (such as grade 3 , deep myometrial invasion, LVSI, and lymph node involvement) is highest in the p53-abn group. Type II endometrial cancer (uterine carcinosarcoma, uterine serous carcinoma, and clear cell carcinomas) patients have a higher P53-abn than endometrioid carcinoma, even grade 3 endometroid endometrial cancer patients. The prognosis for these patients was generally poor [19]. MMR-d group and POLE-mt group showed a similar prevalence of grade 3 and endometrioid histotype. The prevalence of parameters of aggressiveness (LVSI-positive and deep myometrial invasion ) was lower in the POLE-mt group than the MMR-d group. In particular, the POLE-mt group was the only group that showed a null prevalence of lymph node involvement (0\%). No endometrioid subtypes of these two groups have been described such as clear cell and mixed endometrioid/clear cell carcinomas [20-22]. These findings support the exceptionally favorable prognosis of the POLE-mt group. The second analysis of the PORTEC-1 and PORTEC-2 cohort found that fewer recurrences and a lower risk for cancer-specific death in patients with grade 3 POLE-mt tumors, compared with $30.9 \%$ of other grade 3 tumors within that cohort [23]. The majority of grade 1-2 endometrial cancers were classified into the p53-wt group and parameters of aggressiveness of this type is low. Therefore, since the prognosis of the p53-wt group varies from good to moderate [24]. The Disease spread beyond the uterus is seldom seen in high-grade endometrial cancer harboring POLE-mt. Thus, simple hysterectomy and bilateral salpingo-oophorectomy maybe an appropriate selection. POLE-mt did not increase sensitivity to radiotherapy nor chemotherapeutics in mouse-derived embryonic stem cells. These results support studies exploring the minimization of adjuvant therapy for early-stage POLE-mt endometrial cancers [25]. In contrast, more aggressive surgery seems appropriate for women with p53abn endometrial cancers. Molecular classification of grade 3endometrial cancer reveals that these tumors are a mixture of molecular subtypes of endometrial carcinoma, rather than a homogeneous group [26]. Our study was in concordance with the above results which underlying the great need for molecular-driven clinical trials to stratify the patients into a better therapy modality on type of hysterectomy and adjuvant RT.

Our study did not acquire any information on tumor recurrence or exact details, which could have helped to investigate differences in progression-free survival. Data from large-scale trials and prospective multicentered studies are needed because of the rarity of Stage II EC in a single center.

\section{Conclusions}

This study revealed that in patients stage II EC, radical hysterectomy was associated with shorter OS and CSS than TH combined with vaginal BT in patients with high-risk EC.

\section{Declarations}

\section{Authors Contribution}


Dr. Wu proposed the concept and designed the study. Drs Wang and Ran contributed to the acquisition of data. Dr Wang performed the statistics. Dr Wang interpreted the data and wrote the manuscript at the help of Ran. Dr. Wu performed critical revision of the manuscript and addressed the comments from the journal.

\section{Compliance with ethical standards}

Conflict of interest: All authors declare that they have no conflict of interest.

Ethics approval and consent to participate: All procedures performed in studies involving human participants were in accordance with the ethical standards of the institutional and/or national research committee and with the 1964 Helsinki declaration and its later amendments or comparable ethical standards. This study was approved by the Ethic Committee (EC) from the Beijing Gynecology and Obstetrics Hospital. All data involving in this study come from the SEER registry.

Availability of data and material: The datasets generated and analyzed during the current study are available in the SEER database.

Funding: There is no funding support for this study.

Informed consent: Informed consent was obtained from all individual participants included in the study

\section{References}

1. Siegel RL, Miller KD, Jemal A (2018) Cancer statistics. CA: a cancer journal for clinicians 68:7-30.

2. Chen WQ, Sun KX, Zheng RS, Zhang SW, Zeng HM, Xia CF, et al (2018) Cancer incidence and mortality in China,2014. Chinese journal of cancer research 30:1-12.

3. The American College of Obstetrics and Gynecologists (2014) Morcellation and Occult Malignancy in Gynecologic Surgery. Available at: http://www.sgo.org/wpcontent/uploads/2014/04/ACOG_Statement.pdf.

4. ACOG practice bulletin, clinical management guidelines for obstetrician-gynecologists (2005): management of endometrial cancer. American College of Obstetricians and Gynecologists 105:413425.

5. Boente MP, Yordan EL Jr, Mclntosh DG, Grendys EC Jr, Orandi YA, Davies S, et al (1993) Prognostic factors and long-term survival in endometrial adenocarcinoma with cervical involvement. Gynecol Oncol 51:316-322.

6. Sartori E, Gadducci A, Landoni F, Lissoni A, Maggino T, Zola P, et al (2001) Clinical behavior of 203 stage II endometrial cancer cases: the impact of primary surgical approach and of adjuvant radiation therapy. Gynecol Cancer 11:430-437.

7. Koh WJ, Abu-Rustum NR, Bean S, Bradley K, Campos SM, Cho KR, et al (2018) Uterine neoplasms, version 1.2018, NCCN clinical practice guidelines in oncology. J Natl Compr Canc Netw 16:170-99 
8. Liu T, Tu H, Li Y, Liu Z, Liu G, Gu H (2019) Impact of Radical Hysterectomy Versus Simple Hysterectomy on Survival of Patients with Stage 2 Endometrial Cancer. A Meta-analysis. Ann Surg Oncol 26:2933-2942.

9. National Cancer Institute Surveillance (2019) Epidemiology, and End Results Program. Cancer statistics Available at: http://seer.cancer.gov/.

10. Lewin, Sharyn N (2011) Revised FIGO staging system for endometrial cancer. J Clinical Obstetrics \& Gynecology 54:215-218.

11. Wright JD, Fiorelli J, Kansler AL, Burke WM, Schiff PB, Cohen CJ. et al (2009) Optimizing the management of stage II endometrial cancer. the role of radical hysterectomy and radiation. Obstet Gynecol 200:419e1-419e7.

12. Leminen A, Forss M, Lehtovirta P. (2011) Endometrial adenocarcinoma with clinical evidence of cervical involvement: accuracy of diagnostic procedures, clinical course, and prognostic factors. Acta Obstet Gynecol Scand 74:61-66.

13. Takano M, Ochi H, Takei Y, Miyamoto M, Hasumi Y, Kaneta Y,et al (2013) Surgery for endometrial cancers with suspected cervical involvement: is radical hysterectomy needed (a GOTIC study)? Br J Cancer 109:1760-1765.

14. Nout RA, van de Poll-Franse LV, Lybeert ML, Wárlám-Rodenhuis CC, Jobsen JJ, Mens JW, et al (2011) Long-term outcome and quality of life of patients with endometrial carcinoma treated with or without pelvic radiotherapy in the post operative radiation therapy in endometrial carcinoma 1 (PORTEC-1) trial. J Clin Oncol 29:1692-1700.

15. Keys HM, Roberts JA, Brunetto VL, Zaino RJ, Spirtos NM, Bloss JD, et al (2004) A phase III trial of surgery with or without adjunctive external pelvic radiation therapy in intermediate risk endometrial adenocarcinoma: a Gynecologic Oncology Group study. Gynecol Oncol 92:744-751.

16. Eltabbakh GH, Moore AD (1999) Survival of women with surgical stage Il endometrial cancer. Gynecol Oncol 74:80-85.

17. Ayhan A, Taskiran C, Celik C, Yuce K.(2004) The long-term survival of women with surgical stage II endometrioid type endometrial cancer. Gynecol Oncol 93:9-13.

18. Raffone A, Travaglino A, Mascolo M, Carotenuto C, Guida M, Mollo A, et al (2020) Histopathological characterization of ProMisE molecular groups of endometrial cancer. Gynecol Oncol. 157:252-259.

19. Zhang C, Hu W, Jia N, Li Q, Hua K, Tao X, et al (2015) Uterine carcinosarcoma and high-risk endometrial carcinomas: a clinicopathological comparison. Int J Gynecol Cancer 25:629-636.

20. DeLair DF, Burke KA, Selenica P, Lim RS, Scott SN, Middha S, et al (2017) The genetic landscape of endometrial clear cell carcinomas. J Pathol 243:230-241.

21. Rosa-Rosa JM, Leskelä S, Cristóbal-Lana E, Santón A, López-García MÁ, Muñoz G, et al (2016) Molecular genetic heterogeneity in undifferentiated endometrial carcinomas. Mod Pathol 29:1594.

22. Köbel M, Tessier-Cloutier B, Leo J, Hoang LN, Gilks CB, Soslow RA, et al (2017) Frequent mismatch repair protein defificiency in mixed endometrioid and clear cell carcinoma of the endometrium. Int $\mathrm{J}$ Gynecol Pathol 36:555-561 
23. Stelloo E, Nout RA, Osse EM, Jürgenliemk-Schulz IJ, Jobsen JJ, Lutgens LC, et al (2016) Improved Risk Assessment by Integrating Molecular and Clinicopathological Factors in Early-stage Endometrial Cancer-Combined Analysis of the PORTEC Cohorts. Clin Cancer Res 22:4215-4224.

24. Raffone A, Travaglino A, Mascolo M, Carbone L, Guida M, Insabato L, et al (2019) TCGA molecular groups of endometrial cancer. Pooled data about prognosis. Gynecol Oncol 155:374-383.

25. Van Gool IC, Rayner E, Osse EM, Nout RA, Creutzberg CL, Tomlinson IPM, et al (2018) Adjuvant Treatment for POLE Proofreading Domain-Mutant Cancers: Sensitivity to Radiotherapy, Chemotherapy, and Nucleoside Analogues. Clin Cancer Res 24:3197-3203.

26. Bosse T, Nout RA, McAlpine JN, McConechy MK, Britton H, Hussein YR, et al (2018) Molecular Classification of Grade 3 Endometrioid Endometrial Cancers Identifies Distinct Prognostic Subgroups. Am J Surg Pathol 42:561-568.

\section{Figures}

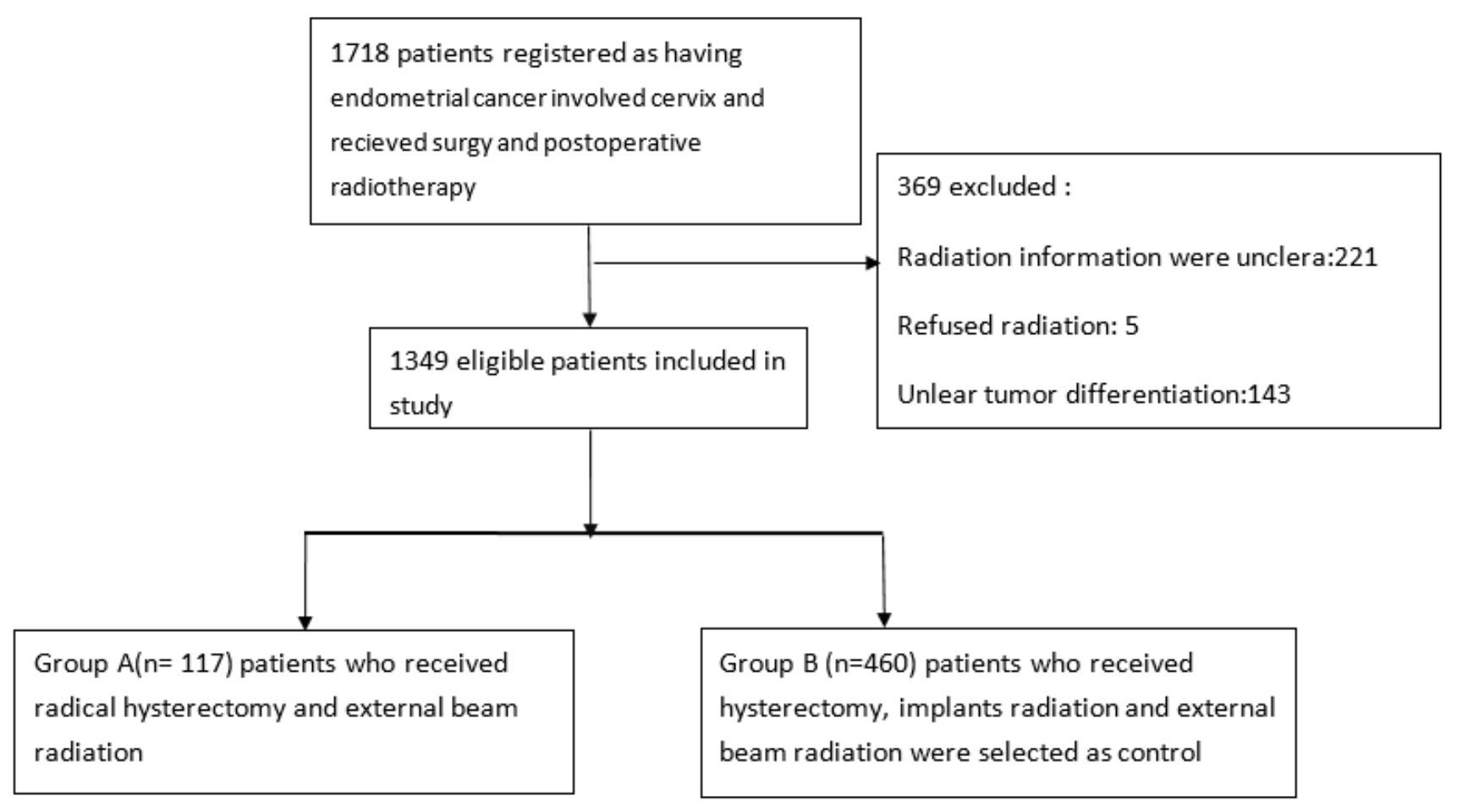

Figure 1

Patients selections and study design 


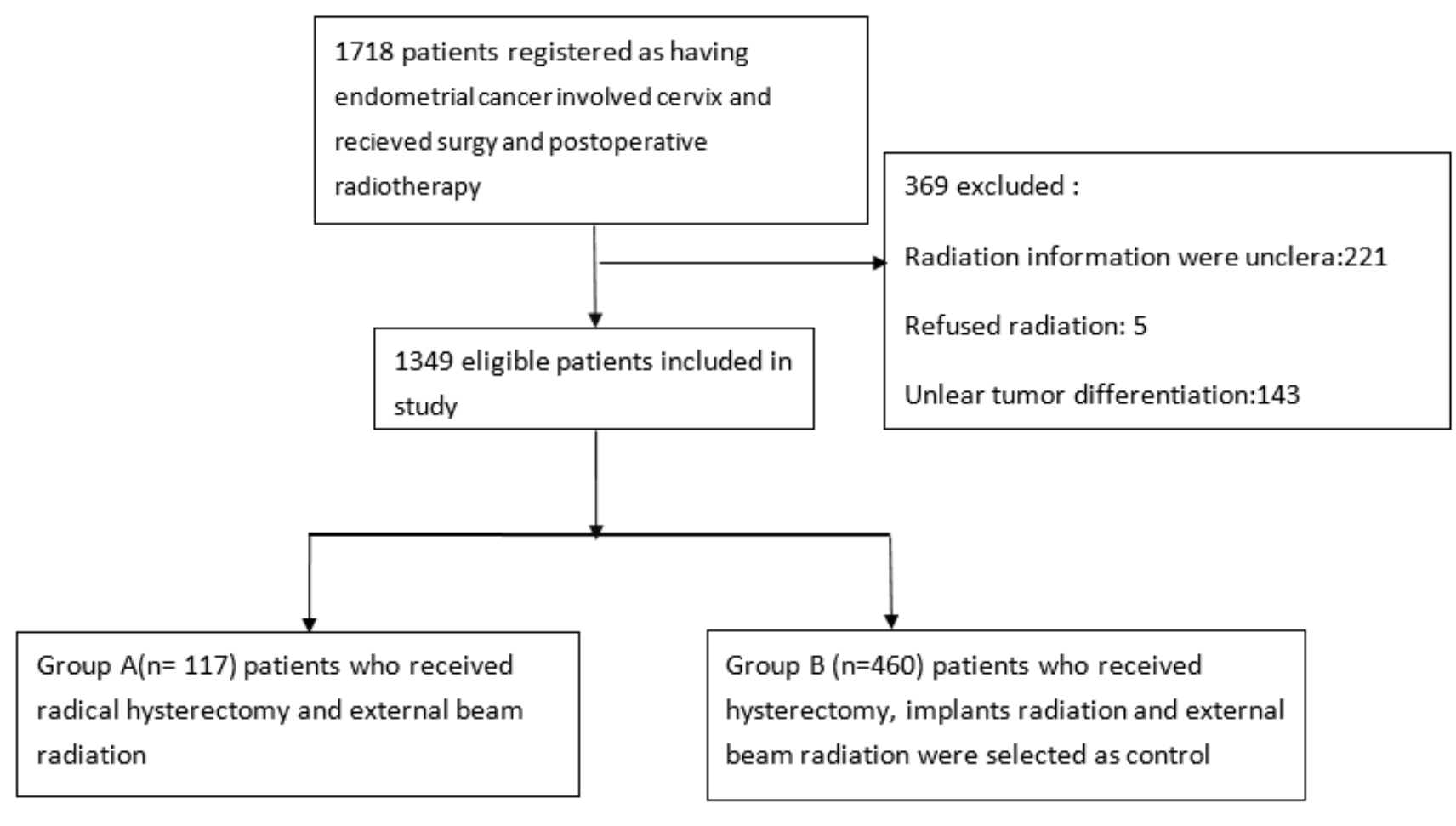

Figure 1

Patients selections and study design
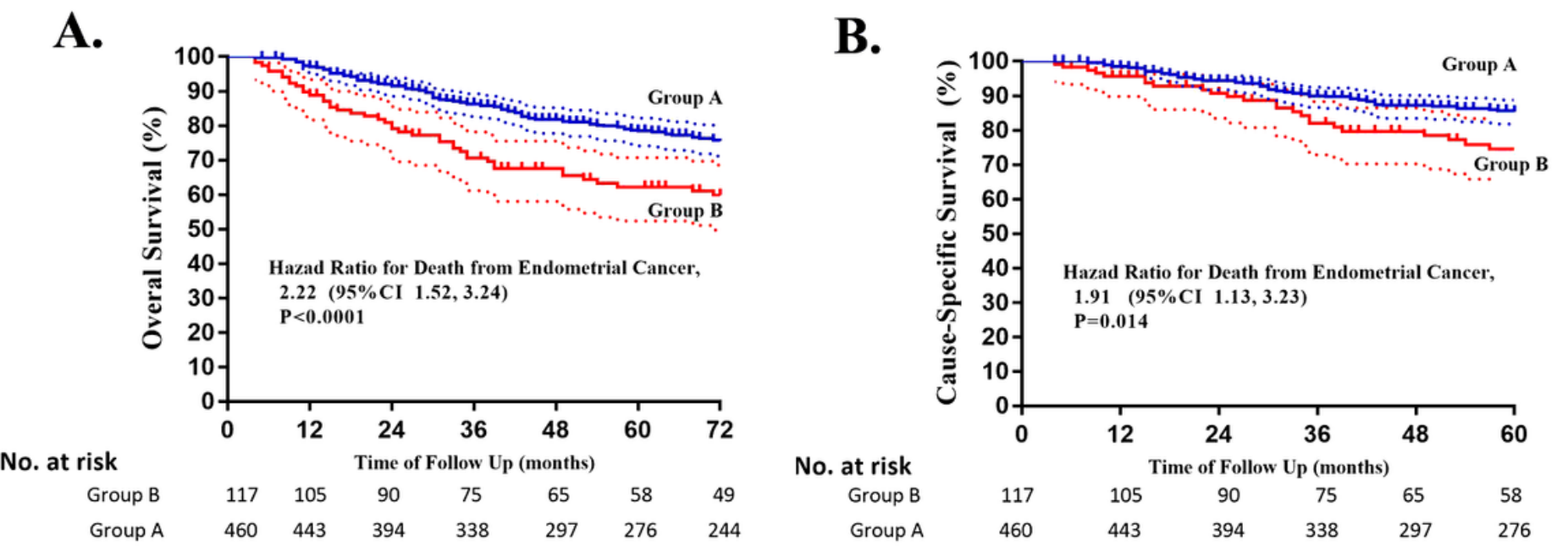

Figure 2

Kaplan-Meier Estimates of Overall Survival and Cause-specific Survival. (A) shows the Kaplan-Meier plot for overall survival in patients with Stage II Endometrial Cancer who received Radical Hysterectomy or Simple Hysterectomy and Brachytherapy. (B) shows the cause-specific survival in Patients with Stage II Endometrial Cancer who received Radical Hysterectomy or Simple Hysterectomy and Brachytherapy. A Cox proportional hazards model was used to determine the hazard ratio and $95 \%$ confidence interval. Tick marks indicate censored data. 

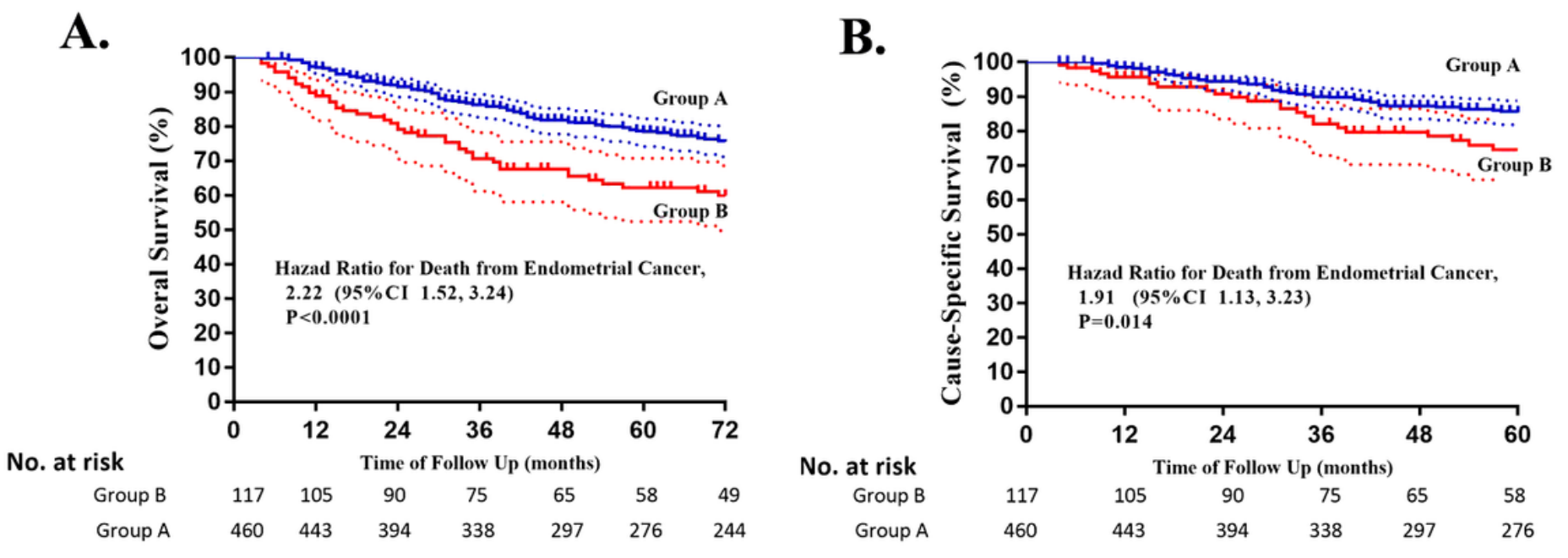

\section{Figure 2}

Kaplan-Meier Estimates of Overall Survival and Cause-specific Survival. (A) shows the Kaplan-Meier plot for overall survival in patients with Stage II Endometrial Cancer who received Radical Hysterectomy or Simple Hysterectomy and Brachytherapy. (B) shows the cause-specific survival in Patients with Stage II Endometrial Cancer who received Radical Hysterectomy or Simple Hysterectomy and Brachytherapy. A Cox proportional hazards model was used to determine the hazard ratio and $95 \%$ confidence interval. Tick marks indicate censored data.
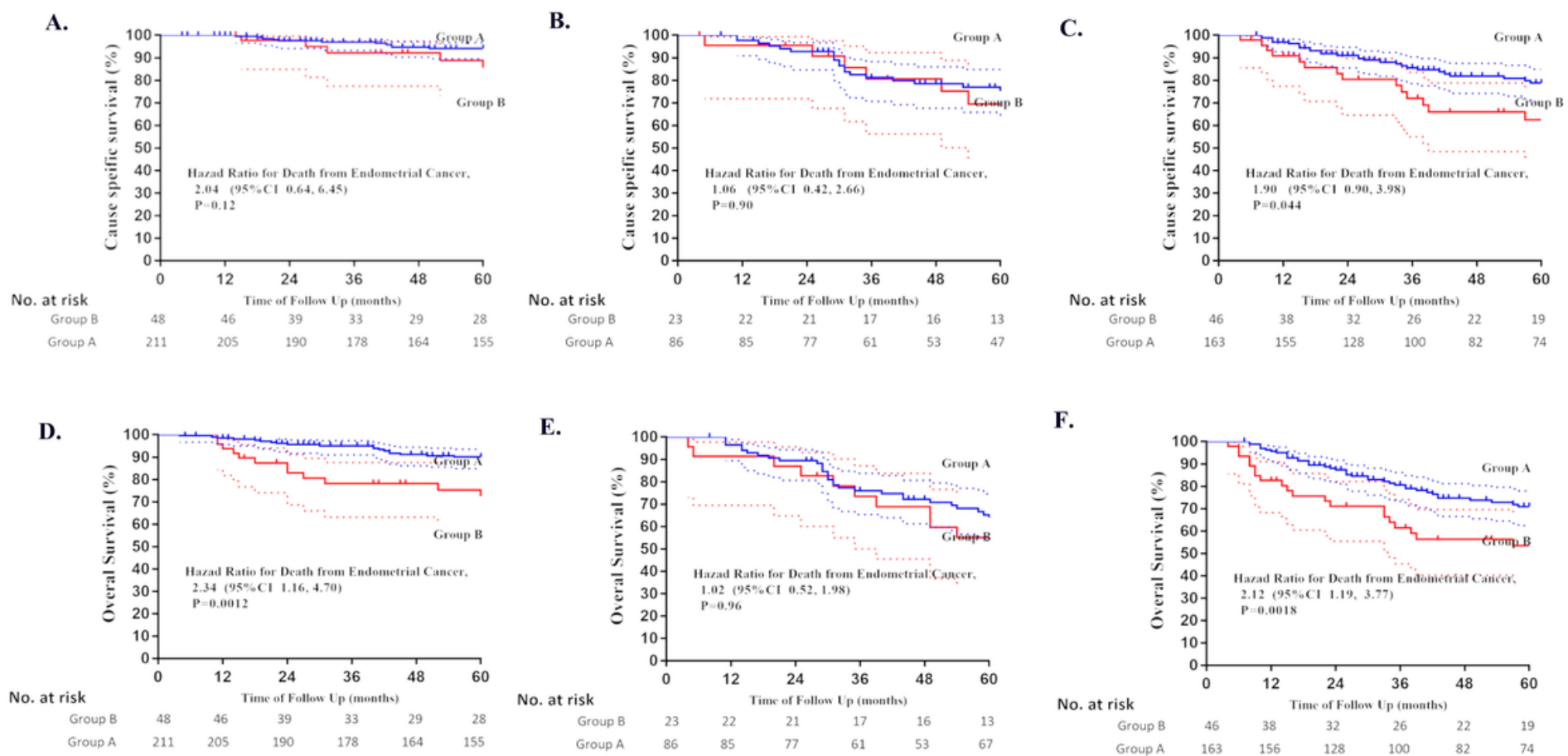

Figure 3 
Kaplan-Meier Estimates of Overall Survival and Cause-specific Survival in Patients with different disease differentiation. (A) shows the Kaplan-Meier plot for cause-specific survival in patients with grade 3 lowrisk EC; (B) shows the Kaplan-Meier plot for cause-specific survival in patients with grade 1-2 low-risk EC; (C) shows the Kaplan-Meier plot for cause-specific survival in patients with high-risk EC; (D) shows the Kaplan-Meier plot for overall survival in patients with grade 3 low-risk EC; (E) shows the Kaplan-Meier plot for overall survival in patients with grade 1-2 low-risk EC; (F) shows the Kaplan-Meier plot for overall survival in patients with high-risk EC.
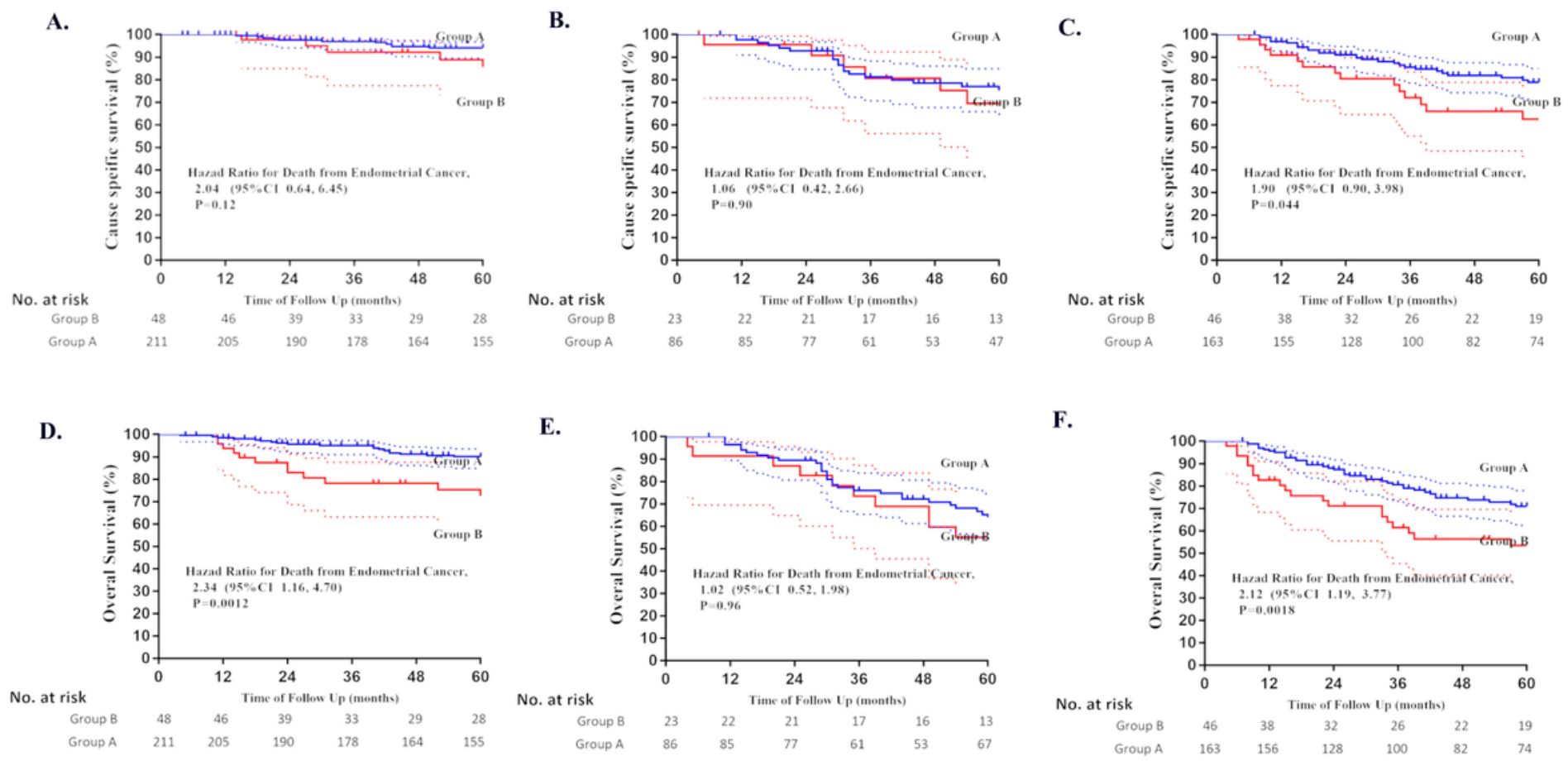

\section{Figure 3}

Kaplan-Meier Estimates of Overall Survival and Cause-specific Survival in Patients with different disease differentiation. (A) shows the Kaplan-Meier plot for cause-specific survival in patients with grade 3 lowrisk EC; (B) shows the Kaplan-Meier plot for cause-specific survival in patients with grade 1-2 low-risk EC; (C) shows the Kaplan-Meier plot for cause-specific survival in patients with high-risk EC; (D) shows the Kaplan-Meier plot for overall survival in patients with grade 3 low-risk EC; (E) shows the Kaplan-Meier plot for overall survival in patients with grade 1-2 low-risk EC; (F) shows the Kaplan-Meier plot for overall survival in patients with high-risk EC. 